\title{
Evolução Temporal da Mortalidade por Cancro do Colo do Útero em Portugal
}

\section{Time-Trends in Cervical Cancer Mortality in Portugal}

Cristina TEIXEIRA $\square^{1,2}$, Ana Maria PEREIRA², Eugénia ANES ${ }^{2,3}$, Carina RODRIGUES ${ }^{2,3}$, Maria José CASTANHEIRA ${ }^{2}$

Acta Med Port 2019 Jun;32(6):427-433 - https://doi.org/10.20344/amp.8921

\section{RESUMO}

Introdução: A mortalidade por cancro do colo do útero em Portugal apresenta valores mais elevados em relação a outros países europeus. O objetivo deste estudo é avaliar a variação da mortalidade por cancro do colo do útero, observada em Portugal nas últimas seis décadas.

Material e Métodos: Obtivemos taxas de mortalidade por cancro do colo do útero (padronizadas para a idade) reportadas em Portugal (1955-2014), através da International Agency for Research on Cancer. Utilizando análise de regressão linear joinpoint, obtivemos a percentagem de variação anual da taxa (\%VA) e respetivo intervalo de confiança a 95\% (IC 95\%) de acordo com a idade (30-39; 40-49, 50-64; 65-74 e $\geq 75$ anos)

Resultados: No grupo com 30-39 anos, a mortalidade por CCU diminuiu 1,9\% ao ano, durante todo o período (IC 95\%: -2,3; -1,4), atingindo 0,5/100 $000 \mathrm{em}$ 2014. Nas mulheres com 40-49 anos houve decréscimo acentuado entre 1971 e 1981 (\%VA = -11,6; IC 95\%: $-14,6 ;-8,6)$, com subsequente aumento de $2,4 \%$ ao ano (IC $95 \%$ : 1,0; 3,8) até 2001, mas que reverteu a partir desse ano $(\% \mathrm{VA}=-5,2$; IC 95\%: $-7,7 ;-2,6)$, atingindo $3,0 / 100000$ em 2014. A mortalidade por cancro do colo do útero diminuiu de 29,2 para $6,7 / 100000$, de 34,3 para $7,7 / 100000$, e de 24,7 a $9,2 / 100000$, respetivamente, nas mulheres com $50-64,65-74$ e com 75 ou mais anos. Nestes três grupos o decréscimo ocorreu principalmente nas décadas de 70 e 80 , não havendo variação significativa da taxa nas últimas três décadas.

Discussão: O maior decréscimo da mortalidade por cancro do colo do útero observada em Portugal ocorreu na década de 70 em paralelo com profundas alterações no Sistema Nacional de Saúde caracterizadas pelo aumento do acesso ao diagnóstico precoce e da qualidade do tratamento. Nas últimas três décadas, a mortalidade por cancro do colo do útero estabilizou em idades mais avançadas, o que pode ser explicado parcialmente por fatores com impacto na adesão aos programas de rastreio.

Conclusão: Houve marcado declínio da mortalidade por cancro do colo do útero em todos os grupos etários, embora com estagnação deste indicador desde meados da década de 80 para grupos etários mais avançados. Os resultados sugerem a necessidade de incrementar a adesão a programas de rastreio em Portugal.

Palavras-chave: Neoplasias do Colo do Útero/mortalidade; Mortalidade/tendências; Portugal

\section{ABSTRACT}

Introduction: The mortality rate due to cervical cancer is higher in Portugal compared to other European countries. This study aimed to evaluate the time-trends in cervical cancer mortality rates observed in Portugal over the last six decades.

Material and Methods: Age-standardized cervical cancer mortality rates reported in Portugal between 1955 and 2014 , were collected from the International Agency for Research on Cancer (IARC). Joinpoint regression analysis was used to identify significant changes in mortality rates by assessing the percentage of annual variation ( $\% \mathrm{AV})$ of the rate and respective $95 \%$ confidence interval $(95 \% \mathrm{Cl})$ according to the age groups.

Results: Among women with 30-39 years, cervical cancer mortality decreased $1.9 \%$ per year $(95 \% \mathrm{Cl}:-2.3 ;-1.4)$ throughout the time-period, reaching 0.5/100 000 in 2014. Among women aged 40-49 years, CC mortality decreased between 1971 and 1981 $(\% \mathrm{AV}=-11.6$; IC 95\%: $-14.6 ;-8.6)$. Rates then increased by $2.4 \%$ per year $(95 \% \mathrm{Cl}: 1.0 ; 3.8)$ until 2001 and such trend reverted from 2001 onwards (\%AV $=-5.2$; IC 95\%: -7.7; -2.6), reaching 3.0/100 000 in 2014. In women aged 50-64, 65-74 and 75 years or older, cervical cancer mortality rates decreased from 29.2 to $6.7 / 100000$, from 34.3 to 7.7/100 000 and from 24.7 to 9.2/100 000. The decline in mortality rates in these three age groups occurred mainly between 1970 and 1980, and there have been no significant changes in the last three decades.

Discussion: In Portugal, the most impressive decline in cervical cancer mortality rates occurred in the 1970s concurrently with changes in the National Healthcare System. The most important changes were the increased access to early diagnosis and the improvement in therapeutic approaches. The plateau that we observed among older women over the last three decades can be partially explained by factors with negative impact on adherence to cervical screening.

Conclusion: There was a marked decrease in mortality due to CC among all age groups. However, we observed a plateau of this indicator in more advanced age groups over the last three decades. These findings suggest the need of promoting adherence to cervical screening in Portugal.

Keywords: Mortality/trends; Portugal; Uterine Cervical Neoplasms/mortality

\section{INTRODUÇÃO}

Na Europa, o cancro do colo do útero (CCU) é a oitava neoplasia mais frequente nas mulheres, correspondendo a $3 \%$ dos novos casos de cancro estimados em 2018. Embo- ra haja uma marcada discrepância entre áreas geográficas, o CCU constitui uma importante causa de morte por cancro na população feminina em alguns países europeus. ${ }^{1}$ Por cada 100 óbitos devidos a tumores malignos registados

1. Unidade de Investigação em Epidemiologia. Instituto de Saúde Pública. Universidade do Porto. Porto. Portugal.

2. Instituto Politécnico de Bragança. Escola Superior de Saúde. Bragança. Portugal.

3. Núcleo de Investigação e Intervenção no Idoso. Bragança. Portugal.

$\triangle$ Autor correspondente: Cristina Teixeira. cristina.teixeira@ipb.pt

Recebido: 03 de março de 2017 - Aceite: 09 de janeiro de 2019 | Copyright @ Ordem dos Médicos 2019 
em 2014 na população feminina portuguesa, dois foram por CCU e quase um quarto dos óbitos por esta neoplasia ocorreram em mulheres com idade inferior a 55 anos. $^{2}$

O desenvolvimento de CCU é o resultado da conjunção de vários fatores que incluem o tabagismo, o uso prolongado de contracetivos hormonais, a multiparidade e a imunossupressão, nomeadamente a resultante da infeção pelo vírus da imunodeficiência humana. ${ }^{3}$ No entanto, o fator determinante da incidência é a infeção sexualmente transmissível pelo vírus do papiloma humano (HPV) que é a causa necessária para desenvolvimento deste cancro. ${ }^{3,4}$

A infeção por HPV é adquirida por via sexual e causa lesões intraepiteliais escamosas de baixo grau que regridem sem intervenção. Em cerca de $10 \%$ dos casos, a infeção por HPV persiste detetável por longo período de tempo, determinando o desenvolvimento de neoplasia intraepitelial cervical grau 2 ou superior. ${ }^{3,4}$ A transição entre as células escamosas e as células colunares secretoras do epitélio de revestimento é a zona do colo uterino com maior risco para transformação neoplásica na sequência de infeção. ${ }^{4}$ Embora possam ocorrer outros subtipos histológicos, cerca de $75 \%$ dos casos de carcinoma invasivo do colo do útero têm origem nas células escamosas. . $^{3,5}$

A deteção precoce de neoplasia intraepitelial cervical de grau 2 ou 3 possibilita a intervenção atempada com recurso à excisão e vigilância mais frequente, que é determinante na prevenção de carcinoma invasivo ${ }^{3,6}$ diminuindo a probabilidade de desenvolver neoplasia invasiva. A citologia cervical é o método de referência para o rastreio primário do CCU. ${ }^{7,8}$ No entanto, existem atualmente técnicas baseadas na biologia molecular para deteção de HPV que permitem identificar grupos de risco elevado ${ }^{3,9}$ e aumentar a sensibilidade do rastreio. ${ }^{10}$

A vacinação em larga escala para prevenção da infeção por HPV faz-se em vários países, ${ }^{3,11}$ mas a sua introdução é ainda muito recente para se observar o seu impacto na incidência e mortalidade por CCU. A introdução de programas de rastreio bem organizados e acessíveis a toda a população, incluindo os testes de deteção do HPV, são importantes estratégias de saúde pública com impacto evidente na redução da mortalidade por CCU.7,11,12

A monitorização da evolução temporal da mortalidade por CCU adquire particular importância em saúde pública, permitindo detetar tendências menos favoráveis, ou até mesmo procurar novas estratégias preventivas, tendo em conta que um reforço da prevenção terá efeito na redução da mortalidade.

Com este estudo pretendemos avaliar a tendência temporal da mortalidade por CCU observada em Portugal entre 1955 e 2014.

\section{MATERIAL E MÉTODOS}

Este estudo consiste numa análise descritiva da evolução temporal da mortalidade por CCU, observada ao longo das últimas seis décadas em Portugal. A informação foi obtida a partir da base de dados da International Agency of Research on Cancer (IARC), ${ }^{13}$ para todos os anos exceto o período de 2004 a 2006 e o ano 2014 para os quais obtivemos informação do Instituto Nacional de Estatística (INE), ${ }^{2}$ por não estarem disponíveis na base IARC. Para cada ano entre 1955 e 2014 obteve-se a taxa de mortalidade por CCU para a população portuguesa, considerando grupos etários de cinco em cinco anos. Para estes grupos etários, avaliámos visualmente o padrão de variação temporal da taxa de mortalidade, de forma a reagrupar grupos etários subsequentes, mas com padrão de variação semelhante. Excluímos da análise idades inferiores a 30 anos, devido ao número limitado de óbitos nestas mulheres. Os grupos etários foram agrupados considerando as seguintes classes: $30-39,40-49,50-64,65-74$ e igual ou superior a 75 anos. A taxa de mortalidade por CCU padronizada para a idade, foi calculada para cada ano em estudo e para cada um destes grupos etários, utilizando o método de padronização direto e a população europeia (1976) como padrão. ${ }^{14}$

Para avaliar a tendência ao longo do tempo da mortalidade por CCU utilizaram-se modelos de regressão linear segmentada (joinpoint regression models) aplicados a cada grupo etário. Esta abordagem estatística permitiu obter segmentos de reta separados por pontos de inflexão (joinpoints) que indicam os anos em que houve mudança significativa na evolução temporal da mortalidade. ${ }^{15} \mathrm{~A}$ partir do declive de cada segmento de reta é possível obter a percentagem de variação anual (\%VA) da taxa. Desta forma, obtivemos medidas de tendência ao longo do tempo expressas em \%VA negativa (se houve diminuição durante o período) ou positiva (se houve aumento durante o período) e respetivo intervalo de confiança a 95\% (IC 95\%). Para esta análise, utilizou-se o programa Joinpoint versão 4.3.1.0. ${ }^{16}$ As taxas de mortalidade observadas e previstas pelo modelo de regressão apresentam-se como sendo o número de óbitos por 100000 mulheres do respetivo grupo etário. O nível de significância considerado foi de 0,05.

Utilizaram-se dados disponibilizados ao público em geral pelo IARC e pelo INE, por isso, não houve necessidade de aprovação pela Comissão de Ética.

\section{RESULTADOS}

Em Portugal, entre 1955 e 2014, a taxa de mortalidade por CCU, padronizada para a idade (população europeia como padrão, 1976), em mulheres com 30 ou mais anos, diminuiu de 19,4 para 4,4/100 000. O decréscimo da taxa foi evidente para todas as idades, mas o padrão de variação deste indicador é diferente de acordo com o grupo etário considerado. A Fig. 1 apresenta a variação das taxas de mortalidade observadas e previstas (de acordo com os resultados obtidos pelo modelo de regressão) ao longo do período em estudo. A Tabela 1 apresenta a \% VA desta taxa, respetivo IC 95\% e os pontos de inflexão, com alteração significativa da tendência temporal, para cada grupo etário.

Nas mulheres mais jovens a taxa de mortalidade padronizada diminuiu de 3,7 para 0,5 por 100000 mulheres deste grupo etário (Fig. 1). Este decréscimo foi constante (sem 
pontos de inflexão) e significativo ao longo do período em estudo, com uma \% VA de -1,9 (IC 95\% entre -2,3 e-1,4) tal como se apresenta na Tabela 1.

Nas mulheres com idade entre 40 e 49 anos, a taxa de mortalidade padronizada para a idade diminuiu ao longo do período em estudo de 17,4 para 3,0/100 000 (Fig. 1), com pontos de inflexão em 1971, 1981 e 2001 (Tabela 1). Apenas entre 1981 e 2001 a tendência inverteu observando-se um aumento significativo de $2,4 \%$ ao ano (IC 95\% entre 1,0 e 3,8$)$ tal como se observa na Tabela 1.

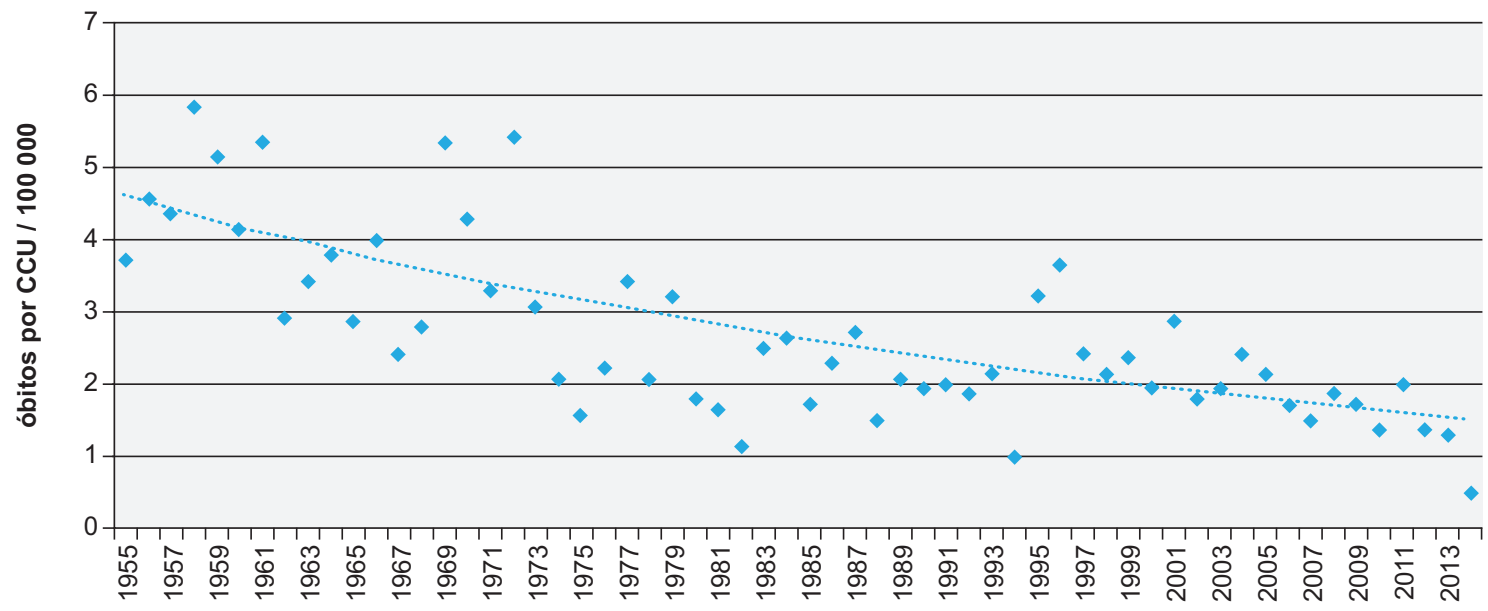

observado 30-39 anos

previsto 30-39 anos
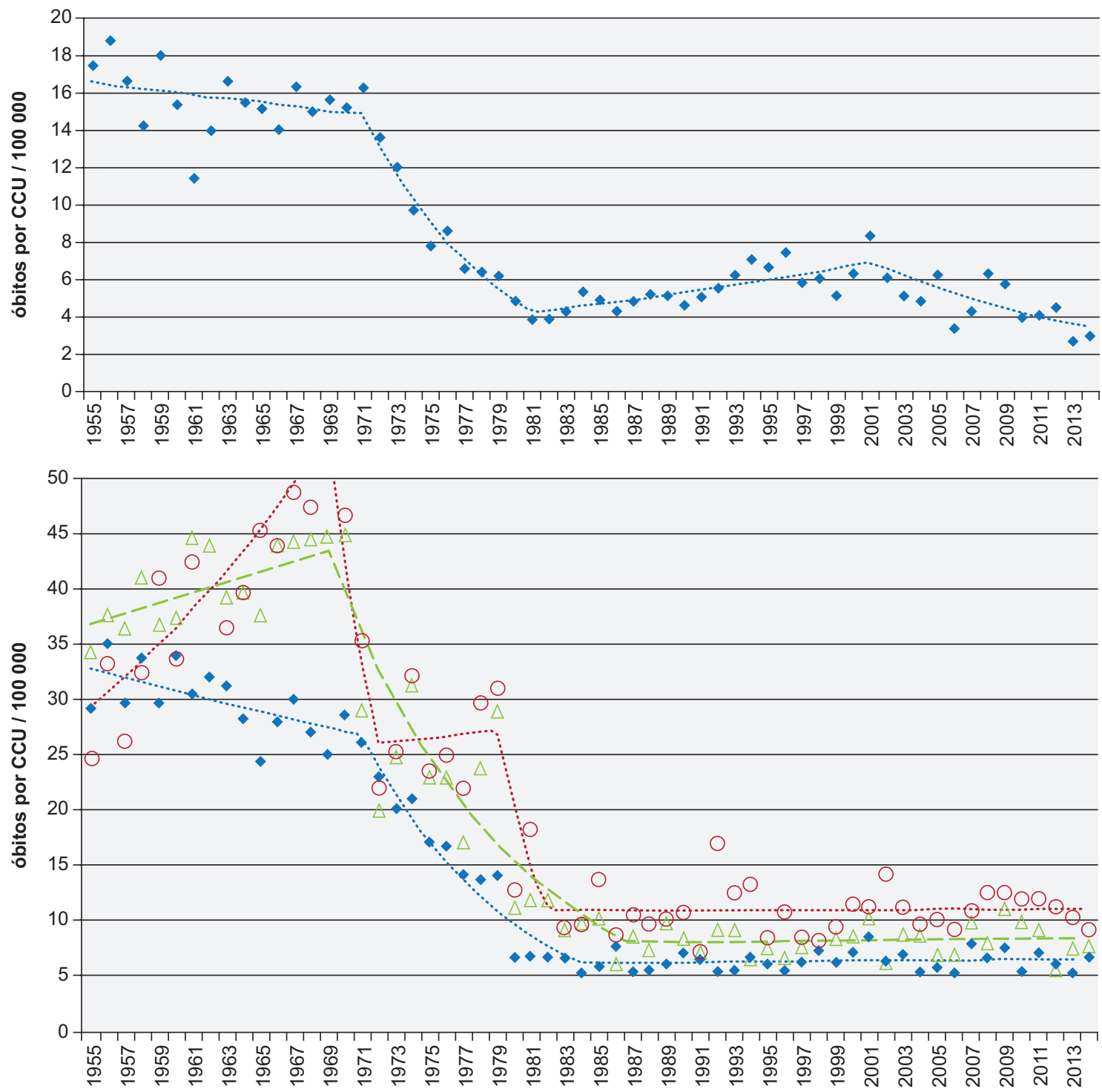

. previsto 40-49 anos

observado 40-49 anos (10

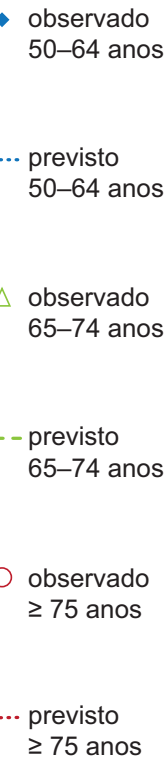

Figura 1 - Evolução da mortalidade por CCU, padronizada de acordo com a idade; Portugal 1955-2014 
Entre 1955 e 2014 a mortalidade por CCU variou de 29,2 a $6,7 / 100000$, de 34,3 a 7,7/100 000 e de 24,7 a 9,3/100 000, em mulheres com 50-64, 65-74 e 75 ou mais anos, respetivamente (Fig. 1). Nas primeiras três décadas da série temporal, a taxa de mortalidade por CCU nas mulheres com idade entre 50 e 64 anos apresentou um decréscimo significativo entre 1955 e 1984. No grupo etário entre 65 e 74 anos o decréscimo foi significativo entre 1969 e 1987. Nas mulheres mais velhas, a taxa de mortalidade por CCU apresentou um aumento significativo entre 1955 e 1969 e tendências decrescentes entre 1969 e 1982. Os três grupos com idade mais avançada apresentam padrão similar da variação da taxa de mortalidade por $\mathrm{CCU}$, verificando-se ausência de alterações significativas desde 1984, 1987 e 1982, respetivamente nos grupos entre 50-64, 65-74 e com 75 ou mais anos, tal como se apresenta na Tabela 1.

\section{DISCUSSÃO}

Entre 1955 e 2014 houve redução evidente da mortalidade por CCU na população portuguesa. Embora tenha havido uma redução da mortalidade por $\mathrm{CCU}$ em todos os grupos etários ao longo do período em estudo, verificou-se que o decréscimo foi constante em mulheres mais jovens enquanto que em grupos de idade mais avançada verificase uma estagnação da taxa nos últimos anos da série.

Portugal é um dos países da Europa Ocidental que tem apresentado as mais elevadas taxas de mortalidade por CCU. Esta superioridade relativamente a outras populações europeias foi particularmente evidente antes da década de 70 , altura em que houve um marcado declínio deste indicador em Portugal, aproximando os valores da mortalidade por CCU entre Portugal e outros países europeus. Contudo, a estagnação destes valores observada desde
1982, colocou Portugal novamente no grupo de países da Europa Ocidental com valores mais elevados de mortalidade por esta forma de cancro. ${ }^{1}$ Esta situação preocupante observada em Portugal já foi reportada por outros autores $^{17,18}$ e merece particular atenção.

O presente estudo envolve dados recolhidos ao longo de seis décadas. Diferenças na forma de diagnóstico de CCU e de registo da causa de morte ao longo do tempo podem influenciar as tendências temporais da taxa de mortalidade ${ }^{19}$ Ao longo do período em estudo houve alterações no sistema de classificação internacional de doenças (CID), tendo havido três atualizações deste sistema, desde 1970, sendo a mais recente a $10^{\text {a }}$ revisão. ${ }^{20-22}$ De acordo com os resultados deste estudo, houve uma marcada mudança das tendências temporais da taxa de mortalidade, precisamente na década de 70 . É pouco provável que a variação tão marcada desta taxa possa dever-se exclusivamente a alterações da CID. Além disso, a variação da taxa não foi uniforme nos cinco grupos etários em análise.

A par com outros países da Europa, no primeiro quartel do século XX, surgiu em Portugal o primeiro centro oncológico com localização na capital. ${ }^{23}$ Ao longo dos anos subsequentes, os serviços oncológicos oferecidos por este centro desenvolveram-se com constante reorganização logística e tecnológica. ${ }^{24}$ No final da década de 50 e durante as décadas de 60 e 70 houve expansão e descentralização dos serviços oncológicos em Portugal com a abertura de novos centros, primeiro em Coimbra e mais tarde no Porto. Em simultâneo, houve considerável melhoria das tecnologias e abordagens para o tratamento do cancro, bem como a implementação de rastreio com o intuito de diagnóstico precoce de lesões pré-invasoras suspeitas. ${ }^{23,24}$ Durante a década de 70, Portugal assistiu a uma profunda

Tabela 1 - Percentagem de variação anual (\%VA) para a taxa de mortalidade por cancro do colo uterino

\begin{tabular}{|c|c|c|c|c|}
\hline $\begin{array}{c}\text { Grupo Etário } \\
\text { (anos) }\end{array}$ & Pontos de inflexão & Período de tempo & $\%$ VA [IC 95\%] & Valor-p \\
\hline $30-39$ & & $1955-2014$ & $-1,9[-2,3 ;-1,4]$ & $<0,001$ \\
\hline \multirow{4}{*}{$40-49$} & & $1955-1971$ & $-0,6[-1,6 ; 0,4]$ & 0,208 \\
\hline & 1971 & $1971-1981$ & $-11,6[-14,6 ;-8,6]$ & $<0,001$ \\
\hline & 1981 & $1981-2001$ & $2,4[1,0 ; 3,8]$ & 0,001 \\
\hline & 2001 & $2001-2014$ & $-5,2[-7,7 ;-2,6]$ & $<0,001$ \\
\hline \multirow{3}{*}{$50-64$} & & $1955-1971$ & $-1,2[-2,1 ;-0,4]$ & 0,004 \\
\hline & 1971 & $1971-1984$ & $-10,7[-12,5 ;-8,8]$ & $<0,001$ \\
\hline & 1984 & $1984-2014$ & $0,2[-0,5 ; 0,9]$ & 0,534 \\
\hline \multirow{3}{*}{$65-74$} & & $1955-1969$ & $1,2[-0,4 ; 2,8]$ & 0,148 \\
\hline & 1969 & $1969-1987$ & $-9,0[-10,6 ;-7,3]$ & $<0,001$ \\
\hline & 1987 & $1987-2014$ & $0,2[-1,1 ; 0,5]$ & 0,787 \\
\hline \multirow{5}{*}{$\geq 75$} & & $1955-1969$ & $4,5[2,9 ; 6,1]$ & $<0,001$ \\
\hline & 1969 & $1969-1972$ & $-21,7[-42,8 ; 7,0]$ & 0,121 \\
\hline & 1972 & $1972-1979$ & $0,7[-5,5 ; 7,4]$ & 0,819 \\
\hline & 1979 & $1979-1982$ & $-26,2[-55,7 ; 23,1]$ & 0,238 \\
\hline & 1982 & $1982-2014$ & $-0,0[-0,8 ; 0,9]$ & 0,935 \\
\hline
\end{tabular}


mudança no sistema de saúde, particularmente no acesso aos cuidados de saúde. Em 1971 foi legislada a reforma do sistema de saúde e assistência com o estabelecimento dos Centros de Saúde como esboço do futuro Serviço Nacional de Saúde (SNS), o qual foi implementado em 1979. Apesar da limitada implementação, os primeiros centros de saúde desempenharam funções essencialmente de prevenção, associadas à promoção e vigilância da saúde ${ }^{25,26} \mathrm{~A}$ conjugação destes fatores terá contribuído para o decréscimo da mortalidade por CCU observado na década de 70 na população portuguesa, maior facilidade de acesso ao diagnóstico precoce, e seu tratamento e melhoria da qualidade do mesmo, resultando num aumento da sobrevivência. Este aumento, como consequência do diagnóstico precoce e melhoria no tratamento do CCU, foi reportado para outra população europeia, embora tal efeito tivesse ocorrido quase duas décadas mais cedo em relação a Portugal. ${ }^{27}$

Um estudo que se reporta aos primeiros anos do milénio mostra que a incidência de CCU tem diminuído em Portugal, mas a taxa de mortalidade padronizada não mostrou tendência decrescente entre 1980 e 2005. ${ }^{17}$ De acordo com os nossos resultados, a estagnação da mortalidade por CCU manteve-se nos últimos 30 anos, em mulheres de idade mais avançada. Em Espanha foram reportados resultados semelhantes. ${ }^{28}$

O exame citológico para deteção de CCU tem forte impacto na redução da mortalidade devido a esta forma de neoplasia. , $^{3,8} \mathrm{Em}$ Portugal esta forma de diagnóstico surgiu na década de $60,{ }^{29}$ mas foi exclusivamente utilizada para rastreios de forma oportunista durante a maior parte do período de tempo em análise. Embora tenha havido no final da década de 60 iniciativas de rastreio organizado para o $\mathrm{CCU}$, esta atividade ocorreu em áreas muito restritas da capital portuguesa. ${ }^{23} \mathrm{~A}$ implementação de rastreios organizados de base populacional teve início apenas em 1990 na região Centro e só a partir de 2008 se difundiu esta prática a outras regiões de Portugal. ${ }^{30}$ Além disso, a taxa de cobertura geográfica destes rastreios é inferior a 50\%, com taxas de adesão variável de acordo com a região, mas que globalmente apresentaram tendência decrescente nos últimos quatro anos atingindo um valor próximo de $50 \%$ em $2014 .^{30}$ Esta situação pode ser apenas o reflexo da utilização de rastreio oportunista, no contexto de procura de cuidados de saúde ginecológicos, ou na sequência de consultas de planeamento familiar, desviando as mulheres dos rastreios de base populacional. No entanto, tal situação pode ser explicada pela não adesão a qualquer tipo de rastreio. Em Portugal, ${ }^{31,32}$ à semelhança do observado em Espanha, ${ }^{28}$ tem sido demonstrada uma menor prevalência de adesão ao exame citológico cervical em mulheres de idade mais avançada em comparação com as mais jovens.

Esta situação poderá contribuir para a estagnação das taxas de mortalidade por CCU em idades mais avançadas.

De acordo com Wang et al, a ocorrência de CCU, em mulheres de idade avançada, depende do grau de adesão ao rastreio entre os 50 e 60 anos de idade havendo maior probabilidade de CCU nas que não foram adequadamen- te rastreadas. ${ }^{33}$ Tem sido também reportado que a atrofia epitelial e a regressão da zona de transformação observadas após a menopausa determinam menor efetividade do rastreio com o avançar da idade. ${ }^{34}$ Além disso, a infeção pelo HPV apresenta um estado de latência, mantido sob controlo imunológico na célula epitelial basal (germinativa), podendo haver uma reativação periódica da infeção numa idade mais avançada. ${ }^{35}$ Tendo em conta que a sobrevivência após CCU diminui à medida que aumenta a idade, ${ }^{36}$ a conjugação destas circunstâncias pode explicar a estagnação das taxas de mortalidade em idades mais avançadas. Por conseguinte, há necessidade de delinear estratégias de prevenção do CCU direcionadas para mulheres com idade mais avançada.

As mulheres com menos de 40 anos, em que é mais provável a existência de uma gravidez, com eventual contacto com profissionais de saúde no período perinatal, têm maior probabilidade de serem rastreadas. Este aspeto pode explicar a tendência decrescente ao longo de todo o período em análise para a mortalidade por CCU que se observa no grupo etário mais jovem. O padrão de variação da mortalidade de CCU observado na população portuguesa, parece indicar que nos grupos etários mais jovens a redução se deve ao rastreio e a abordagens terapêuticas atempadas.

Em Portugal, a incidência mais elevada de CCU observa-se em mulheres com idade entre 40 e 49 anos. ${ }^{1}$ De acordo com os nossos resultados, nas mulheres desta faixa etária observou-se um aumento da mortalidade por CCU nas décadas de 80 e 90, embora nos últimos anos da série temporal se tenha observado uma inversão desta tendência. Outros autores têm mostrado esta tendência desfavorável explicando-a pelo fluxo migratório proveniente das ex-colónias portuguesas em África que se observou em Portugal desde 1975. ${ }^{18}$ Há maior prevalência de infeção por HPV e de infeção por VIH em populações de países africanos. ${ }^{18,37} \mathrm{~A}$ coexistência de infeção por HPV e $\mathrm{VIH}$ determinam um maior risco de mortalidade por $\mathrm{CCU}^{7}$ nestas populações.

Relativamente à incidência de $\mathrm{CCU}$, tem sido reportada uma diminuição de carcinoma epidermoide mas um aumento de adenocarcinoma ${ }^{38} \mathrm{O}$ adenocarcinoma está associado a pior prognóstico ${ }^{5}$ e o aumento deste subtipo de CCU pode ser explicado pela maior dificuldade de detetar estas lesões através de exame citológico. ${ }^{3,5} \mathrm{~A}$ base de dados utilizada para esta análise não nos permite averiguar o contributo de cada subtipo histológico de CCU na mortalidade, o que constitui uma limitação deste estudo. Outros estudos são necessários para avaliar a incidência de adenocarcinoma e o seu contributo na mortalidade por CCU na população portuguesa.

Tem sido demonstrada uma diminuição da incidência de lesões do colo do útero em áreas geográficas onde foi introduzida a vacina contra o HPV. ${ }^{39} \mathrm{~A}$ vacinação foi introduzida em Portugal há uma década, ${ }^{29}$ pelo que se aguardam estudos para perceber o seu efeito a longo prazo na mortalidade por CCU no nosso país. Entretanto, deve haver incentivos à participação em rastreios na população 
portuguesa, aconselhando e promovendo a adesão aos rastreios de toda a população feminina.

\section{CONCLUSÃO}

Houve marcado declínio da mortalidade de CCU em Portugal em todos os grupos etários analisados. No entanto, o valor deste indicador mantém-se praticamente inalterado desde meados da década de 80 , para todos os grupos etários, excetuando o grupo mais jovem. Estes resultados sugerem que deve haver investimento em transmitir informação relevante à população portuguesa, com o intuito de aumentar a sua participação em rastreios para CCU.

\section{PROTECÇÃO DE PESSOAS E ANIMAIS}

Os autores declaram ter utilizado dados disponibilizados ao público em geral pelo IARC e pelo INE, pelo que não houve necessidade de aprovação pela Comissão de Ética.

\section{REFERÊNCIAS}

1. International Agency for Research on Cancer. Global Cancer Observatory. [consultado 2016 jul 6]. Disponível em: http://www-dep. iarc.fr/WHOdb/WHOdb.htm.

2. Instituto Nacional de Estatística. Base de Dados: Saúde, Mortalidade por causas de morte. [consultado 2016 jul 6]. Disponível em: https://www.ine.pt/xportal/xmain?xpid=INE\&xpgid=ine_indicadores \&indOcorrCod=0005052\&contexto=bd\&selTab=tab2.

3. Small W, Bacon MA, Bajaj A, Chuang LT, Fisher BJ, Harkenrider MM, et al. Cervical cancer: a global health crisis. Cancer. 2017;123:2404-12.

4. Gravitt PE, Winer RL. Natural history of HPV infection across the lifespan: role of viral latency. Viruses. 2017;9:1-10.

5. Fujiwara H, Yokota H, Monk B, Treilleux I, Devouassoux-Shisheboran M Davis A, et al. Gynecologic cancer InterGroup (GCIG) consensus review for cervical adenocarcinoma. Int J Gynecol Cancer. 2014;24:S96-101.

6. Silva DP da, Moutinho JM. Sociedade Portuguesa de Ginecologia. Secção Portuguesa de Colposcopia e Patologia Cervico-Vulvovaginal. Consenso em Patologia Cervico-Vulvovaginal. 2004. [consultado 2016 jul 6]. Disponível em: http://www.spginecologia.pt/uploads/patologia.pdf.

7. Schiffman M, Wentzensen N, Wacholder S, Kinney W, Gage JC, Castle $\mathrm{PE}$. Human papillomavirus testing in the prevention of cervical cancer. J Natl Cancer Inst. 2011;103:368-83.

8. Smith RA, Brooks D, Cokkinides V, Saslow D, Brawley OW. Cancer screening in the United States, 2013: a review of current American Cancer Society guidelines, current issues in cancer screening, and new guidance on cervical cancer screening and lung cancer screening. CA Cancer J Clin. 2013;63:88-105.

9. Goodman A. HPV testing as a screen for cervical cancer. BMJ. 2015;350:h2372.

10. Dijkstra MG, Snijders PJ, Arbyn M, Rijkaart DC, Berkhof J, Meijer CJ. Cervical cancer screening: on the way to a shift from cytology to full molecular screening. Ann Oncol. 2014;25:927-35.

11. Bosch FX, Broker TR, Forman D, Moscicki AB, Gillison ML, Doorbar J, et al. Comprehensive control of human papillomavirus infections and related diseases. Vaccine. 2013;31:S1-31.

12. Peirson L, Fitzpatrick-Lewis D, Ciliska D, Warren R. Screening for cervical cancer: a systematic review and meta-analysis. Syst Rev. 2013;2:35.

13. World Health Organization. Cancer mortality data base. Lyon, France: Internantional Agency for Research on Cancer. [consultado 2016 jul 6]. Disponível em: http://www-dep.iarc.fr/WHOdb/WHOdb.htm.

14. EUROSTAT.Revision of the European Standard Population. Report of Eurostat's task force. 2013. [consultado 2016 dez 29]. Disponível em: http://ec.europa.eu/eurostat/documents/3859598/5926869/KS-RA-13028-EN.PDF/e713fa79-1add-44e8-b23d-5e8fa09b3f8f.

15. Bastos J, Barros $\mathrm{H}$, Lunet $\mathrm{N}$. Evolução da mortalidade por cancro da mama em Portugal (1955-2002). Acta Med Port. 2007;20:139-44.

16. National Cancer Institute: Statistical Research and Applications Branch.

\section{CONFIDENCIALIDADE DOS DADOS}

Os autores declaram ter seguido os protocolos do seu centro de trabalho acerca da publicação de dados.

\section{CONFLITOS DE INTERESSE}

Os autores declaram não ter conflitos de interesses relacionados com o presente trabalho.

\section{FONTES DE FINANCIAMENTO}

Este trabalho não recebeu qualquer tipo de suporte financeiro de nenhuma entidade no domínio público ou privado.

Joinpoint Trend Analysis Software, version 4.3.1.0. [consultado 2016 abr 20]. Disponível em: https://surveillance.cancer.gov/joinpoint/.

17. Alves C, Alves L, Lunet N. Epidemiology of cervical cancer. Arq Med. 2010;24:266-77.

18. Pinheiro PS, Tyczynsky J, Bray F, Amado J, Matos E, Miranda AC, et al Cancro em Portugal. Lyon: IARC Technical Publication; 2002.

19. Janssen $F$, Kunst AE. ICD coding changes and discontinuities in trends in cause-specific mortality in six European countries, 1950-99. Bull World Health Organ. 2004; 82:904-13.

20. Diário do Governo. n. ${ }^{\circ} 79 / 1970,1{ }^{\circ}$ Suplemento, Série I de 1970-04-04. Decreto-Lei 138/70. 1970. [consultado 2016 dez 29]. Disponível em: https://dre.pt/application/file/a/219874

21. Diário da República n. ${ }^{\circ} 297 / 1979,3^{\circ}$ Suplemento, Série I de 1979-12-27. Decreto-Lei n. ${ }^{\circ}$ 513-C1/79. 1979. [consultado 2016 dez 29]. Disponível em: https://dre.pt/application/file/a/147370.

22. Ministério do Equipamento, do Planeamento e da Administração do Território. Conselho Superior de Estatística. Deliberação n. ${ }^{\circ} 131 / 97$ de 27 de Julho. 1997. [consultado 2016 dez 29]. Disponível em: http:// portalcodgdh.min-saude.pt/index.php/Aprova\%C3\%A7\%C3\%A3o da_CID-10_para_utiliza\%C3\%A7\%C3\%A3o_no_Sistema_Estat $\%$ C $\overline{3}$ $\%$ ADstico Nacional.

23. Serviço Nacional de Saúde. Instituto Português de Oncologia de Lisboa [consultado 2017 jan 20]. Disponível em: http://www.ipolisboa.minsaude.pt/Default.aspx?Tag=DOSSIER\&Dossierld=6.

24. Costa RM. Luta contra o cancro e oncologia em Portugal. Estruturação e normalização de uma área científica. Centro de Investigação Transdisciplinar Cultura Espaço Memória. Edições Afrontamento. 2012. [consultado 2017 jan 20]. Disponível em: http://ler.letras.up.pt/uploads/ ficheiros/10411.pdf.

25. Branco AG, Ramos V. Cuidados de saúde primários em Portugal. Rev Port Saúde Pública. 2001;2:5-12.

26. Sakellarides C, Reis V, Escoval A, Conceição C, Barbosa P. O futuro do Sistema de Saude Português: "Saúde 2015". Lisboa: Escola Nacional de Saúde Pública, UNL; 2005.

27. Sparen P, Gustafsson L, Friberg LG, Ponten J, Bergstrom R, Adami HO. Improved control of invasive cervical cancer in Sweden over six decades by earlier clinical detection and better treatment. J Clin Oncol. 1995;13:715-25.

28. Cervantes-Amat M, Lopez-Abente G, Aragones N, Pollan M, PastorBarriuso R, Perez-Gomez B. The end of the decline in cervical cancer mortality in Spain: trends across the period 1981-2012. BMC Cancer. 2015;15:287.

29. Direção Geral de Saúde. Comissão Técnica de Vacinação. Programa Nacional de Vacinação (PNV). Introdução da vacina contra infecções por Vírus do Papiloma Humano. Texto de apoio à Circular Normativa n. ${ }^{\circ} 22$ /DSCS/DPCD de 17 de Outubro de 2008. [consultado 2017 jan 20]. Disponível em: http://www.spdc.pt/files/legix/11245_3.pdf. 
30. Miranda N, Portugal C, Dinis A, Roldão M, Matias A. Serviço Nacional de Saúde. Rede Nacional de Especialidade Hospitalar e de Referenciação de Radioterapia. 2a Revisão. 2015. [consultado 2017 jan 20]. Disponível em: http://www2.acss.min-saude.pt/Portals/0/RelatorioFinal Radioterapia.pdf.

31. Alves C, Alves L, Lunet N. Prevalence and determinants of cervical cytology use in an urban sample of Portuguese women. Eur $\mathrm{J}$ Cancer Prev. 2009;18:482-8.

32. Oliveira M, Peleteiro B, Lunet N. Cytology use for cervical cancer screening in Portugal: results from the 2005/2006 National Health Survey. Eur J Public Health. 2014;24:253-8.

33. Wang J, Andrae B, Sundström K, Ploner A, Ström P, Elfström KM, et al. Effectiveness of cervical screening after age 60 years according to screening history: nationwide cohort study in Sweden. PLoS Med. 2017;14:1-17.

34. Schlichte M, Guidry J. Current Cervical Carcinoma Screening Guidelines. J Clin Med. 2015;4:918-32.
35. Maglennon GA, McIntosh PB, Doorbar J. Immunosuppression facilitates the reactivation of latent papillomavirus infections. J Virol. 2014; 88:710-6.

36. Ioka A, Tsukuma $\mathrm{H}$, Ajiki W, Oshima A. Influence of age on cervical cancer survival in Japan. Jpn J Clin Oncol. 2005;35:464-9.

37. CPLP/ONUSIDA. EpidemiadeVIHnospaíses delíngua oficial portuguesa. 2010. [consultado2018 out22]. Disponívelem: https://www.cplp.org/Files/ Filer/VIH\%20em\%20pa\%C3\%ADses\%20de\%20L\%C3\%ADngua\%20 Portuguesa \%20-\%202\%C2\%AA\%20Edi\%C3\%A7\%C3\%A3o\%20$\% 20$ FINAL.pdf.

38. van der Horst J, Siebers AG, Bulten J, Massuger LF, de Kok IM Increasing incidence of invasive and in situ cervical adenocarcinoma in the Netherlands during 2004-2013. Cancer Med. 2017;6:416-23.

39. Hestbech MS, Lynge E, Kragstrup J, Siersma V, Vazquez-Prada Baillet $M$, Brodersen J. The impact of HPV vaccination on future cervical screening: a simulation study of two birth cohorts in Denmark. BMJ Open. 2015;5:e007921. 